\title{
ABREUGRAFIA SISTEMATICA EM MASSA: INVIABILIDADE ECONÔMICA E EVENTUAIS PERIGOS DA EXPOSIÇÃO A RADIAÇõES *
}

\author{
Febus Gikovate ** \\ Diogo Pupo Nogueira ***
}

RSPU-B/302

\begin{abstract}
Gikovate, F. \& Nogueira, D. P. - Abreugrafia sistemática em massa: inviabilidade econômica e eventuais perigos da exposição a radiações. Rev. Saúde públ., S. Paulo, 10:103-10, 1976.

RESUMo: E descrita a descoberta, por Manoel de Abreu, da roentgenfotografia e sua aplicação maciça na população brasileira onde muitas vezes, por exigência legal, crianças, jovens, mulheres grávidas eram submetidos a exames repetidos e, frequentemente, desnecessários. Com as explosões atômicas de Hiroshima e Nagasaki e o conhecimento de que mesmo doses minimas de radiações podem condicionar sérios riscos somáticos e genéticos, a abreugrafia sistemática passou a constituir séria preocupação para os sanitaristas, uma vez que as vantagens, que são poucas, oferecidas por este método diagnóstico são contrabalançadas pelo seu alto custo e pelo risco que oferece às populaçóes. Com base na literatura e em particular em declaração específica da Organização Mundial de Saúde, é proposta a abolição da abreugrafia sistemática, com a eliminação, na legislaçäo brasileira, dos dispositivos que a tornam obrigatória em numerosas eventualidades.
\end{abstract}

UNITERMos: Abreugrafia em massa. Radiações.

Em fins de 1936, Manoel de Abreu apresentou à Sociedade Brasileira de $\mathrm{Tu}$ berculose o processo de roentgenfotografia para cadastro torácico. No Hospital Alemão do Rio de Janeiro já estava funcionando um aparelho pioneiro desde junho de 1936, onde foram feitos os ensaios iniciais. Foi nomeada uma comissão constituída por Arlindo de Assis, Genésio Pitanga, Hamilton Nelson e Vitor Cortes, que apresentou em 2 de dezembro do mesmo ano um relatório consagrando o novo processo como método de investigação da tuberculose nas grandes massas ${ }^{1}$.

0 método de fotografia da imagem do ecran radioscópico teve vários precursores. Só chegou a resultados práticos com os estudos de Manoel de Abreu iniciados em 1924. Sob sua orientação, foram fabricados pela Casa Lohner do Rio de Janeiro os primeiros aparelhos.

Em face das conclusões da Comissão designada pela Sociedade Brasileira de

* Trabalho do Instituto de Pneumologia da Santa Casa, do Departamento de Cirurgia da Faculdade de Ciências Médicas da Santa Casa de São Paulo e do Departamento de Saúde Ambiental da Faculdade de Saúde Pública da USP.

* Da Faculdade de Ciências Médicas da Santa Casa de São Paulo. Rua Dr. Cesário Mota Jr., 112 - São Paulo, SP - Brasil.

** Da Faculdade de Saúde Pública da USP - Av. Dr. Arnaldo, 715 - São Paulo, SP — Brasil. 
GIKOVATE, F. \& NoGuEIRA, D. P. - Abreugrafia sistemática em massa: inviabilidade econômica e eventuais perigos da exposição a radiações. Rev. Saúde públ., S. Paulo, 10:103-10, 1976.

Tuberculose, a Saúde Pública, dirigida por J. Barros Barreto e J. P. Fontenelle, instalou o segundo aparelho de abreugrafia em Centro de Saúde 3. Em março de 1937 iniciou-se o cadastro torácico pela abreugrafia em massa.

Estava o Brasil então em plena fase epidêmica no que se refere à tuberculose. Os coeficientes de mortalidade eram, em 1940, nas capitais dos Estados, de 284,6 por cem mil habitantes, segundo dados do Serviço Nacional de Tuberculose (SNT) ? Os recursos terapêuticos estavam então limitados ao chamado regime higieno-dietético em Sanatório e à colapsoterapia médica e cirúrgica, realizada em pacientes internados ou não. Os resultados do tratamento eram em regra bons na tuberculose mínima e raramente satisfatórios nas formas avançadas. 0 dignóstico precoce, através do cadastro torácico, permitiria alcançar a eliminiação precoce dos focos contagiantes através do tratamento que só era realmente eficaz na fase inicial da moléstia. 0 entusiasmo despertado pela descoberta de Manoel de Abreu era então plenamente justificado.

Descobertos os raios X por Roentgen em 1895 e logo aplicados em larga escala em Medicina para fins de diagnóstico e tratamento, surgiram em breve as primeiras vítimas - os manipuladores dos mesmos. Com o aperfeiçoamento dos aparelhos, da técnica e das medidas de proteção, o problema da injúria aguda pelas radiaçōes foi superado.

$O$ conhecimento da ação nociva das radiações em geral, e dos Raios $\mathrm{X}$ e análogos usados na prática médica, só resultou dos estudos e observações feitas durante a $2 .^{a}$ guerra mundial e dos anos subseqüentes. A explosão da bomba atômica em Hiroshima e Nagasaki permitiu o estudo dos efeitos imediatos e remotos das radiações sobre as populações atingidas e sobre as gerações subseqüentes. $\mathbf{O}$ arsenal atômico crescente com as inúmeras explosões experimentais e a utilização em escala cada vez maior da energia atômica para fins pacíficos, alertaram toda a humanidade para os perigos das radiaçōes. O exagero foi inevitável. Em relação à utilização de armas atômicas, as previsões mais pessimistas se justificam. Cabe realizar todos os esforços a fim de evitá-la. Quanto a acidentes devidos à utilização da energia atômica para fins pacíficos, é possível prevení-los.

Do estudo do efeito das radiações sobre a população em geral, devemos destacar as seguintes conclusões: "É possível que a exposição a qualquer quantidade de radiações produza algum efeito biológico. Não há dados que indiquem a dose abaixo da qual não ocorrem certos tipos de efeitos tardios das radiações. As radiações provocadas pelas provas atômicas são pequenas em comparação com a exposição médica em países altamente industrializados" ${ }^{11}$.

"A maior fonte atual de exposição da população a radiações resulta do seu uso na arte médica. Esta constatação nos obriga não só ao manejo ótimo do equipamento existente, mas também à pesquisa ininterrupta com a finalidade de melhorar a eficiência da exposição, de permitir uma radiação de exposição compatível com a obtenção de informação bio-médica necessária. Uma educação adequada especializada de todos que manejam as fontes de radiações na filosofia e nos métodos de proteção às radiações, é um fator no enfoque preventivo da injúria das radiaçōes" ${ }^{15}$.

Entre nós, esses ensinamentos são ignorados, subestimados ou negados por muitos dos que se utilizam das radiaçōes.

Entretanto, Bitteli ${ }^{4}$, em seu Manual de Higiene das Radiações, estuda exaustivamente o problema. Ensina que recomendações gerais, destinadas a reduzir os riscos das radiaçōes já foram feitas no "Radiological Hazards to Patients, Second Report to the Medical Research Council, 1960". Destacaremos apenas duas: 1) De- 
GIKOVATE, F. \& NOGUEIRA, D. P. - Abreugrafia sistemática em massa: inviabilidade econômica e eventuais perigos da exposição a radiações. Rev. Saúde públ., S. Paulo, 10:103-10, 1976.

ve haver indicação clínica precisa antes que seja solicitado qualquer exame radiológico, devendo-se verificar se não existem radiografias anteriores que tornem desnecessária a repetição. 2) Precauções especiais devem ser adotadas para a radiografia de gestantes. Somente exames indispensáveis devem ser realizados e cuidados especiais devem ser tomados a fim de evitar a irradiação do feto. No caso de mulheres em idade procriadora, o clínico deve sempre pensar na possibilidade de uma gravidez em início. Podemos acrescentar - muitas vezes ignorada pela própria paciente.

Entre nós, Maia ${ }^{12}$ vem estudando os efeitos genéticos das radiações no homem, embora os seus trabalhos não tenham a repercussão necessária.

Baikie ${ }^{2}$ chama a atenção para os estudos que detectaram possíveis efeitos crônicos no homem, conseqüência de exposição a doses relativamente baixas de radiaçôes que resultam de radiologia diagnóstica, exposição ocupacional e radioatividade resultante de testes atômicos. Embora os resultados sejam contraditórios, devem ser tomados em consideração. Em relação às fontes de radiações, Baikie afirma que nos Estados Unidos a fonte mais importante é a radiologia médica, que fornece uma dose gonadal média maior do que a radiação natural.

Embora seja difícil evidenciar os efeitos genéticos das radiações médicas, não faltam indicios. A evidência mais direta é o excesso de natimortos e de crianças trissómicas, nascidas de mulheres que foram submetidas a radiografias abdominais antes do parto em questão.

A abreugrafia sistemática feita durante a infância, a puberdade e a idade da procriação indiscriminadamente, sem os cuidados acima citados, representa, sem dúvida, uma dose excessiva de radiaçóes desde que se tome em consideração 0 efeito cumulativo das mesmas. É necessário investigar se o risco assim assumido é compensado por eventuais benefícios que resultariam de um possível diagnóstico mais precoce.

Antes de analisar o problema acima, queremos chamar a atenção para o hábito absurdo e condenável de formar arquivos inúteis de radiografias que obriga os pacientes a repetir várias vezes a mesma radiografia quando encaminhados de um serviço médico para outro. Os prejuízos são óbvios, tanto para a economia dos serviços médicos, como para a saúde dos pacientes. As vantagens são nulas.

O entusiasmo despertado, não só no nosso país, pela descoberta de Manoel de Abreu, encontrou desde o início alguns céticos. Os argumentos apresentados não eram desprovidos de peso e obrigaram Aloisio de Paula a tentar refutá-los. Diziam os céticos que de nada adiantaria descobrir os tuberculosos quando não dispunhamos de meios para isolá-los e tratá-los. Nas décadas de 30 e 40 , tratava-se de uma verdade indiscutível. Não havia leitos suficientes e os resultados do tratamento eram precários. Só mais tarde verificou-se que o método era antieconômico, mesmo quando elevada a prevalência da tuberculose. A pesquisa do B.K. no escarro é, do ponto de vista epidemiológico, mais barata e mais segura, uma vez que elimina os casos de tuberculose inativa e de pneumopatias inespecíficas.

A Organização Mundial de Saúde, no 9. informe do Comitê de Peritos em Tuberculose, em $1974{ }^{13}$, pronunciou-se categoricamente contra a abreugrafia em massa: "O Comitê assinalou que a exploração radiológica em massa com películas de pequenas dimensões (abreugrafia), é um procedimento de detecção de casos muito caro, mesmo quando é elevada a prevalência da tuberculose. Outras desvantagens da exploração radiológica em massa são as seguintes: 1) só contribui 
GIKovate, F. \& NOGUEIRA, D. P. - Abreugrafia sistemática em massa: inviabilidade econômlca e eventuals perigos da exposição a radiaçōes. Rev. Saúde públ., S. Paulo, 10:103-10, 1976.

com uma pequena proporção ao total dos casos detectados; 2) influi pouco sobre a incidência de casos com baciloscopia positiva, já que estes evoluem com tanta rapidez que aparecem entre dois ciclos de exames radiográficos em massa; 3) requer um pessoal médico e técnico muito especializado, que poderia ser mais útil em outras atividades dos serviços sanitários; 4) os aparelhos e veículos usados estão freqüentemente inutilizados por avarias, difíceis de reparar, especialmente quando escasseiam as peças de recâmbio. O Comitê chegou à conclusão de que se devem abandonar os métodos de detecção indiscriminada de casos de tuberculose por meio de equipes móveis de exploração radiológica em massa".

Se analisarmos a experiência brasileira em relação à tuberculose, veremos que as conclusôes são totalmente procedentes. Não temos meios de verificar a soma de recursos gastos com a compra, manutenção e pagamento de técnicos, mas sabemos, com segurança, que foi e continua sendo muito elevada.

0 impacto sobre a mortalidade de tuberculose foi praticamente nulo. Examinando os dados estatísticos do SNT ${ }^{14}$, verificamos que a mortalidade por tuberculose nas capitais estaduais em conjunto passou de 284,6 por cem mil habitantes em 1940 para 241,7 em 1947. A primeira diminuição da mortalidade coincidiu com a introdução da Estreptomicina e PAS, chegando a 121,9 por cem mil habitantes em 1952. Uma segunda diminuição brusca foi devida à introdução da isoniazida no arsenal terapêutico. Assim, em 1953 passou para 81,2 por cem mil habitantes $e$ em 1960 para 69,2 .

0 diagnóstico não se tornou mais precoce. Continuaram a predominar as formas moderada e muito avançada. Ainda em 1973 os pacientes internados no Parque Sanatorial Maria Tereza da Cruzada Bandeirante contra a Tuberculose tiveram a classificação da Tabela $1^{8}$.
TABELA 1

Diagnóstico de pacientes internados no Parque Sanatorial Marla Tereza da Cruzada Bandeirante contra a Tuberculose, em 1973

\begin{tabular}{lr}
\hline Formas avancadas & $280-63,92 \%$ \\
Formas moderadas & $83-18,94 \%$ \\
Formas minimas & $30-6,88 \%$ \\
Outras pneumopatias & $32-7,32 \%$ \\
Sem diagnóstico & $10-2,28 \%$ \\
Outras formas & $03-0,66 \%$ \\
\hline
\end{tabular}

A OMS afirma que a exploração abreugráfica em massa é muito cara mesmo quando a prevalência da tuberculose é elevada. Muito mais cara se torna quando a prevalência é baixa. A título de exemplo mostraremos os resultados obtidos em uma indústria textil de São Paulo. Nas Tabelas 2 e 3 pode-se verificar que a prevalência sempre foi inferior a $1 \%$, oscilando entre 0\% em 1957 e 1975 e $0,65 \%$ em 1955 .

Em 1975 não se detectou nenhum caso de tuberculose. Foram feitas 2563 abreugrafias, 142 foram repetidas e foram necessários 47 exames clínicos e 37 complementares. A baciloscopia dos tossedores é um método mais barato e ainda evita a internação de não tuberculosos e o tratamento específico de pneumopatias não tuberculosas e tuberculoses residuais inativas.

0 exame dos comunicantes PPD positivos deverá ser feito com teleradiografia - menor exposição a radiações e menor número de exames complementares.

O controle do tratamento é fundamentalmente baseado na baciloscopia. O reduzido número de radiografias necessárias no decurso do tratamento não justifica o recurso à abreugrafia. 
GIKOVATE, F. \& NOGUEIRA, D. P. - Abreugrafia sistemática em massa: inviabilidade econômica e eventuais perigos da exposição a radiações. Rev. Saúde públ., S. Paulo, 10:103-10, 1976.

TABELA 2

Resultado de exames de abreugrafia em uma indústria têxtil de Sāo Paulo

\begin{tabular}{|c|c|c|c|c|c|c|c|c|c|c|}
\hline Ano & $\begin{array}{l}\text { Número } \\
\text { de } \\
\text { exames }\end{array}$ & $\begin{array}{l}\text { Imagens } \\
\text { ganglio- } \\
\text { nares }\end{array}$ & $\begin{array}{l}\text { Nódulos } \\
\text { calcifi- } \\
\text { cados }\end{array}$ & Residuais & $\begin{array}{l}\text { Espessa- } \\
\text { mentos } \\
\text { pleurais }\end{array}$ & $\begin{array}{l}\text { Tumores } \\
\text { benignos }\end{array}$ & $\begin{array}{c}\text { Outras } \\
\text { pneumo- } \\
\text { patias }\end{array}$ & \multicolumn{3}{|c|}{$\begin{array}{l}\text { Doentes - T.P. } \\
\text { com B.K. } \\
\text { positivo }\end{array}$} \\
\hline 1952 & 1550 & 7 & 18 & 85 & 6 & 1 & 6 & 1 & - & $0,06 \%$ \\
\hline 1953 & 1577 & 1 & 49 & 73 & 10 & 0 & 8 & 5 & - & $0,31 \%$ \\
\hline 1954 & 1942 & 2 & 43 & 89 & 1 & 1 & 16 & 8 & - & $0.41 \%$ \\
\hline 1955 & 2452 & 0 & 34 & 71 & 1 & 1 & 17 & 16 & - & $0,65 \%$ \\
\hline 1956 & 2203 & 26 & 0 & 71 & 0 & 3 & 19 & & - & $0,18 \%$ \\
\hline 1957 & 750 & 0 & 2 & 36 & 0 & 0 & 6 & & - & $0 \%$ \\
\hline 1958 & 1321 & 0 & 13 & 34 & 0 & 0 & 10 & & - & $0,52 c_{c}$ \\
\hline 1959 & 3395 & 0 & 0 & 175 & 0 & 0 & 11 & 9 & - & $0.26 \%$ \\
\hline 1960 & 2440 & 0 & 0 & 71 & 0 & 0 & 7 & 8 & - & $0,30 \%$ \\
\hline
\end{tabular}

TA B E L A 3

Resultado de abreugrafias em uma indústria têxtil em são Paulo

\begin{tabular}{lr} 
Abreugrafias -1975 & \\
Total de exames & 2563 \\
Normais & 2315 \\
Repetidos & 142 \\
Exame clinico & 47 \\
Exames complementares & 39 \\
Tuberculose & 0 \\
\hline
\end{tabular}

0 controle após tratamento eficaz deverá ser feito durante um a dois anos no máximo, bastando também número reduzido de radiografias.

A OMS ${ }^{13}$ afirma: "As pautas de tratamento são cada vez mais eficazes. as recidivas costumam ser muito raras. $O$ Comitê estima, pois, que agora tem menos importância do que antes a vigilância ulterior dos pacientes que terminaram o tratamento".
Embora óbrio, Baily e col. ${ }^{3}$ se deram ao trabalho de provar com números que o controle radiológico dos pacientes submetidos a quimioprofilaxia com INH é inteiramente dispensável. De 1969 a 1973 todos os pacientes tinham R.X. normal antes e no fim do tratamento. Concluiram que não se deve repetir a radiografia $e$ que o paciente deve ser visto pelo médico uma só rez. Quantas abreugrafias inúteis e. portanto. prejudiciais não são proporcionadas às crianças submetidas a quimioprofilaxia entre nós. nos dispensários e consultórios particulares? E quantas radioscopias em idênticas condições?

Podemos concluir que o uso de radiações pode e deve ser drasticamente reduzido na profilaxia e no tratamento da tuberculose.

As tentativas de conseguir melhorar o diagnóstico precoce do câncer de brônquio. um dos objetiros da abreugrafia. também não deu resultados dignos de nota. Mesmo quando aplicado à população com grande risco, o exame radiológico sistemático não confirmou as esperanças nele depositadas. 
GIKOVATE, F. \& NOGUEIRA, D. P. - Abreugrafia sistemática em massa: inviabilidade econômica e eventuais perigos da exposição a radiações. Rev. Saúde públ., S. Paulo, 10:103-10, 1976.

Fennessy ${ }^{9}$ afirma que: "Embora existam evidências de que o exame radiológico em populaçōes com risco de adquirir câncer do pulmão possa melhorar o prognóstico para alguns pacientes, esta melhora é, na melhor das hipóteses, pequena e, além disso, este fato não é aceito unanimemente como conclusivo". Brett ${ }^{6}$ examinou radiologicamente um grupo de mais de 29.000 pacientes cada 6 meses, durante 3 anos. A idade dos mesmos era acima de 40 anos. Um grupo controle de mais de 25.000 pacientes foi radiografado no início e no fim do estudo. No grupo em estudo, a taxa de sobrevida de 5 anos foi de $15 \%$ e no grupo de controle de $6 \%$. Conclui que houre uma modesta melhora no prognóstico. Não são da mesma opinião Boucot e Weiss ${ }^{5}$, que analisam os resultados do estudo de Brett e mostram que a melhora, na taxa de sobrevida, não é estatisticamente válida. A análise custo/ benefício mostra que há 5 pacientes por dez mil no grupo estudado e 2 no grupo controle. Estimaram o custo de cada um dos 3 casos a mais em, aproximadamente, 83.000 dólares. Assinalaram que se trata de custo mínimo que não tomara em conta a despesa acarretada pelo estudo clínico das pessoas com resultados falsos positivos. Baseando-se em um estudo piloto, feito pela American Cancer Society e pela Veterans Administration. Boucot e Weiss observaram que havia 483 falsos positivos para apenas 97 positivos verdadeiros. Assim, para cada caso positivo verdadeiro havia mais de 4 casos que deriam ser examinados com a finalidade de excluir o diagnóstico de carcinoma. Embora não se possa atribuir um valor monetário a uma "cura" de câncer do pulmão, parece evidente que a radiografia periódica em massa é altamente dispendiosa e de duvidosa eficácia como método de estabelecer diagnóstico "mais precoce" que conduza a mais curas. Wilson ${ }^{16}$ examinon mais de 8 milhões de abreugrafias que provinham de 3 centros. Foram encontrados 906 casos de câncer de pulmão. A sobre- vida destes foi comparada com a de 324 casos de câncer do pulmão diagnosticados em condições habituais de prática clínica. A sobrevida de 5 anos foi de $12.1 \%$ para os provenientes do grupo submetido a abreugrafia sistemática contra $7.9 \%$ para os casos diagnosticados na rotina clínica. Wilson acredita que a radiografia periódica não melhora significativamente $o$ prognóstico do câncer do pulmão.

Fontana e col. ${ }^{10}$ verificaram um aumento de $51 \%$ de sobrevida de 5 anos no projeto da Mayo Clinic. Yeste estudo foram feitas radiografias com intervalo de 4 meses, broncoscopias com flexível e três pesquisas de células neoplásticas no escarro. Trata-se de um estudo piloto, de custo proibitivo. dificilmente aplicável em larga escala, mesmo nos Estados Ĺnidos.

O diagnóstico das afecções cardiovasculares poderá ser feito pela anamnese, medida da pressão arterial e ausculta do coração com mais economia e menos risco para o paciente do que pela abreugrafia.

Somos levados a concluir que os dispositivos * da Consolidação das Leis do Tra-

* Art. 167 - Será obrigatório o exame médico dos empregados por ocasião da admissão, e renovado periodicamente. Nas localidades onde houver serviço de abreugrafia deverá ser utilizado este recurso, na rotina de exames, ao tempo da admissão e todas as vezes em que o mesmo se fizer necessário, a critério médico.

$\$ 10$ - Nas atividades e operaçós insalubres será obrigatório o exame médico periódico dos empregados, de seis em seis meses.

PORTARIA N:0 6, de 26 de janeiro de 1967. Dispōe sobre os trabalhos sob ar comprimido, definidos e caracterizados pela Portaria Ministerial $n \circ 73$.

Art. 2.0 - o exame médico, a que se refere o art. $70^{\circ}$ da aludida Portaria n.073, obedecerá aos seguintes requisitos:

b) radiografia dos pulmōes, coração e vasos da base.

PORTARIA N. 255, de 16 de setembro de 1969. Expede normas de Higiene e Segurança do Trabalho nas minas.

Art. $30^{\circ}$ - E obrigatório o exame de saúde para a admissão de candidato em mina.

$\$ 2.0$ - 0 exame de saúde incluirá radiografia dos pulmóes e do coração. 
GIKOVATE, F. NOGUEIRA, D. P. - Abreugrafia sistemática em massa: inviabilidade econômica e eventuais perigos da exposição a radlações. Rev. Saude públ., S. Paulo, 10:103-10, 1976.

balho deverão ser alterados no sentido de eliminar a exigência indiscriminada de abreugrafias periódicas.

Com mais razão ainda deverá ser eliminada a exigência de abreugrafia para escolares, onde a incidência de tuberculose é muito baixa.

A abolição da abreugrafia, radiografia ou radioscopia não significa, entretanto, abolição do exame médico. Este é indispensável e não será apenas simbólico como é hoje, em face da falsa segurança conferida pelo exame de R.X. A abolição de abreugrafia sistemática foi pleiteada recentemente pelo Prof. Walter Leser, Secretário da Saúde de São Paulo.

A abolição da abreugrafia porá fim ao drama dos portadores de sombras residuais e inativas que só dificilmente conseguem emprego. Será uma medida de economia em material e pessoal técnico e permitirá a sua utilização em outros setores. Ao mesmo tempo será possível reduzir a um mínimo a exposição da população a radiações seguramente não inócuas. Nos casos indicados, as radiaçôes continuarão a ser usadas com as precauçōes ditadas pelo conhecimento de seus efeitos biológicos.

Grkovate, F. \& NogueIRA, D. P. - [Sistematical mass roentgenphotography: economical unviability and eventual danger regarding exposure to radiations]. Rev. Saúde públ., S. Paulo, 10:103-10, 1976.

SUMMARY: The invention of roentgenphotography by Manoel de Abreu and its consequent widespread use in Brasil are described. Due to legal requirements, children, young people and pregnant womem are submitted repeatedly and, very often, to unnecessary examinations. After the atomic explosions of Hiroshima and Nagasaki and the knowledge that even minimum dosis of ionizing radiation can cause serious somatic and genetic risks, the widespread use of abreugraphy became cause of serious concern for Public Health workers, since its advantages are outnumbered by its cost and by the risk it offers to those submitted to this type of examination. According to recent litterature on the subject and following specific recommendations of the World Health Organization, abolition of widespread use of abreugraphy is proposed, with the elimination of the Brazilian legal requirements which make this method of examination mandatory in many situations.

UNITERMS: Abreugraphy. Radiations.

\section{REFERENCIAS BIBLIOGRAFICAS}

1. ABREU, M. \& PAULA, A. - Roentgenfotografia. Rlo de Janeiro, 1940. p. 6.

2. BAIKIE, A. G. - Biological effects of radiation. In: PASSOMORE, R. \& ROBSON, J. S. - A companion to medical studies. Oxford, Blackwell Scientific Publications, 1970. Vol, 2, pp. 33.1.33.8.
3. BAILY, W. C. et al. - Once is enough: chest radiographs for isoniazid recipients. Amer. Rev. resp. Dis., 111: 901-2, 1975.

4. BITELLI, T. - Manual de higiene das radiações e proteção radiológica. São Paulo, Centro de Medicina Nuclear, 1965. (Publlcação CMN 4/65).

5. BOUCOT, K. R. \& WEISS, W. - Is curable lung cancer detected by semi-annual screening? JAMA, 224:1361-5, 1973. 
GIKOVATE, F. \& NoguerRA, D. P. - Abreugrafia sistemática em massa: inviabilidade econômica e eventuais perigos da exposição a radiacoóes. Rev. Saúde públ., S. Paulo, 10:103-10, 1976.

6. BRETT, G. Z. - Earlier diagnosis and survival in lung cancer. Brit. med. $J ., 4: 260-2,1959$.

7. CAMPOS, O. - A tuberculose no Brasil: evolução e tendências. Rev. Serv. nac. Tub., 11:229-42, 1957.

8. CRLZADA BANDEIRANTE CONTRA A TUBERCLLOSE Relatório e Balanço: Dispensario de Santa Cecilia e Parque Sanatorial Maria Tereza. São Paulo, 1973.

9. FENNESSY, M. B. - The radiology of lung cancer. Med. Clin. N. Amer, 59:95-120, 1975

10. FONTANA, R. S. et al. - The mayo Lung project for early detection and localization of bronchogenic carcinoma: a status report. Chest, 67: 511-22, 1975.

11. HEMPELMAN, M.H. - Radiation injury. In: Cecil-Loeb textbook of medicine. 13rd ed. Philadelphia. Saunders, 1971.
12. MAIA, N. F. - Efeitos genéticos das radiações no homem Rev. bras. Radiol., 1:126-32, 1958.

13. ORGANIZACION MUNDIAL DE LA SALUD. Comite de Expertos en Tuberculosis, Ginebra, 1973. Noveno informe. Ginebra, 1974. (Ser. Inf. tecn., 552).

14. RODRIGLES DE ALBUQLERQUE \& VILAS BOAS, A - Informações epidemiológicas sobre a tuberculose nas capitais brasileiras. Rev. Sen. nac. $T u b ., \quad 10: 31-70,1966$.

15. WALD, N $\times$ - Radiation injury In: BEESON, P. B. \& MCDERMOTT, W. Textbook of medicine. 14 a ed. Philadelphia, Saunders, 1975. pp. 66-72.

16. WILSON, H - Prognosis of lung cancer detected in community-wide tuberculosis surveys: an analysis on 906 cases. Med. J. Aust., 23:936-42, 1968 apud FENNESSY

Recebido para publicação em 03/11/1975 A provado para publicaça em $05 / 01 / 1976$ 